\title{
Selecting a Laboratory Information Management System for Biorepositories in Low- and Middle-Income Countries: The H3Africa Experience and Lessons Learned
}

\author{
Samuel Kyobe, Henry Musinguzi, ${ }^{1}$ Newton Lwanga,, Dafala Kezimbira,, Edgar Kigozi, \\ Fred Ashaba Katabazi, Misaki Wayengera,' Moses Lutaakome Joloba,, Emmanuel Akin Abayomi,,3 \\ Carmen Swanepoel, ${ }^{2,3}$ Alash'le Abimiku, ${ }^{4,5}$ Talishiea Croxton,, ${ }^{4,5}$ Petronilla Ozumba, \\ Anazodo Thankgod, ${ }^{5}$ Alan Christoffels, ${ }^{6,7}$ Lizelle van Zyl, Elizabeth Sarah Mayne, \\ Mukthar Kader, Garth Swartz ${ }^{7}$; and H3Africa Biorepository PI Working Group*
}

Biorepositories in Africa need significant infrastructural support to meet International Society for Biological and Environmental Repositories (ISBER) Best Practices to support population-based genomics research. ISBER recommends a biorepository information management system which can manage workflows from biospecimen receipt to distribution. The H3Africa Initiative set out to develop regional African biorepositories where Uganda, Nigeria, and South Africa were successfully awarded grants to develop the state-of-the-art biorepositories. The biorepositories carried out an elaborate process to evaluate and choose a laboratory information management system (LIMS) with the aim of integrating the three geographically distinct sites. In this article, we review the processes, African experience, lessons learned, and make recommendations for choosing a biorepository LIMS in the African context.

Keywords: H3Africa Consortium, LIMS, biorepository, biospecimens, ISBER, genomics

\section{Introduction}

A BIOREPOSITORY REQUIRES the linkage of high-quality material to data housed in a laboratory information management system (LIMS), which tracks each sample. A founding principle of H3Africa is to make sure that DNA (and possibly other biological material) would be properly stored in biorepositories for future research purposes. ${ }^{1,2}$ The value of this material is partly determined by the associated phenotypic data. Management of this data and sample tracking in compliance with national and international best practices and ethical guidelines requires a refined data management system. ${ }^{3,4}$ According to the In- ternational Society for Biological and Environmental Repositories (ISBER) Best Practices, a computer-based inventory system is necessary to track the location and annotation of every specimen in the biorepository. ${ }^{3}$ The system should also track significant events during a sample's existence from collection to destruction, including sample thaws, receipt and/or processing delays, processing, transfer of the sample within the repository, specimen distribution and return, and destruction. ${ }^{3-5}$ These ISBER Best Practices, as well as others from around the world, are under consideration to codify International Organization for Standardization norms as the basis for a new international accreditation program for biorepositories. ${ }^{6}$ There are

\footnotetext{
${ }^{1}$ Makerere University College of Health Sciences, Kampala, Uganda.

${ }^{2}$ Division of Haematology, Department of Pathology, National Health Laboratory Services, Tygerberg Hospital, Cape Town, South Africa.

${ }^{3}$ Division of Haematology, Department of Pathology, Stellenbosch University Faculty of Medicine and Health Sciences, Tygerberg, South Africa.

${ }^{4}$ University of Maryland, College Park, Maryland.

${ }^{5}$ Institute of Human Virology-Nigeria, Abuja, Nigeria.

${ }^{6}$ South African National Bioinformatics Institute, Bellville, South Africa.

${ }^{7}$ University of the Western Cape, Cape Town, South Africa;

${ }^{8}$ Clinical Laboratory Services-Wits Health Consortium, Johannesburg, South Africa.

*The members of the H3Africa Biorepository PI Working Group are listed in the Acknowledgments.
}

(c) Samuel Kyobe et al., 2017; Published by Mary Ann Liebert, Inc. This is an Open Access article distributed under the terms of the Creative Commons Attribution License, which permits unrestricted use, distribution, and reproduction in any medium, provided the original work is properly cited. Mary Ann Liebert, Inc. offers reprint services for those who want to order professionally produced copies of articles published under the Creative Commons Attribution (CC BY) license. To obtain a price quote, email Reprints@ liebertpub.com Please include the article's title or DOI, quantity, and delivery destination in your email. 
several factors that hinder successful implementation of a biorepository LIMS in Africa. This article discusses the $\mathrm{H} 3$ Africa Biorepositories experiences and provides recommendations for the process of evaluating, choosing, and deployment of a sustainable biorepository LIMS in lowand middle-income countries (LMIC).

\section{Materials and Methods}

In 2012 and 2013, the National Institutes of Healthfunded H3Africa Program established three regional biorepositories in Africa in Uganda, South Africa, and Nigeria. The biorepositories were developed to archive and facilitate international scientific access to well-curated biospecimens collected by research projects in the H3Africa Consortium Program. To facilitate international access to biospecimens, it was desirable that the biorepositories set up an interoperable LIMS for data integration and exchange. At the time of funding, each of the three biorepositories had a LIMS for their current capacity. The biorepositories adopted a User LIMS Requirement Checklist provided by Autoscribe Informatics (Berkshire, UK) for the LIMS evaluation. The checklist consists of 15 key user requirements, including, but not limited to, general system information, system configuration and customization, user access and security, functionality, static data tables, reporting system, and system support. Six commercial LIMS companies were invited to respond to the questions and requirements in the evaluation tool. Two open-source LIMS were self-evaluated by the H3Africa biorepositories in Uganda and South Africa. The returned results from three commercial LIMS vendors were combined for analysis and discussed during conference calls and online correspondence. Two biorepository consultants provided advice during the evaluation process. The technical description of how the commercial and open-source LIMS compared with each other is beyond the scope of this article. However, we describe the experience and lessons drawn from the LIMS evaluation process from an African/LIMC perspective.

\section{Results and Discussion}

\section{LIMS selection at the H3Africa biorepositories}

Following the evaluation process, the H3Africa biorepositories made decisions on their biorepository LIMS. The Institute of Human Virology Nigerian-H3Africa Biorepository (I-HAB) in Nigeria decided to upgrade the current LIMS with the latest software updates from the vendor. The Integrated Biorepository of H3Africa Uganda (IBRH $\left.{ }_{3} \mathrm{AU}\right)$ in Uganda decided to acquire a new LIMS and migrate the data from the old platform to the new LIMS. The Clinical Laboratory Services-H3Africa Biorepository (CLS) in South Africa decided to acquire a new LIMS platform in addition to the existing LIMS. Both CLS and $\mathrm{IBRH}_{3} \mathrm{AU}$ chose the same new LIMS platforms. I-HAB was convinced that an upgrade was necessary and sufficient to achieve the interoperable requirements of the three program biorepositories.

\section{Checklist for choosing a LIMS}

The H3Africa project specifications required that the three biorepositories workflows would be harmonized through interoperable LIMS to enable data integration and exchange. Following the evaluation process, the following key ele- ments were considered while choosing a LIMS for the project: (1) customizability and usability; (2) interoperability with other LIMS; (3) access to revisions, updates, patches, and maintenance releases; (4) cost and access to technical support services; (5) maintenance and associated costs; (6) multiuser/site support; (7) robustness to handle large volumes of sample information; (8) security systems (audit trail, user roles, and privileges, etc.); and (9) type of (open source or commercial) LIMS. Table 1 represents a summarized checklist of the elements we considered to be key while choosing a biorepository LIMS. A detailed checklist can be accessed via biorepository.h3africa.org website.

\section{Commercial versus open-source LIMS}

Commercial LIMS are systems whose source codes are developed for sale and need authorization from vendors for licensed use. Open-source LIMS are systems whose source codes are available for distribution at no cost. ${ }^{7}$ During the H3Africa biorepository LIMS evaluation phase, it was decided to implement a commercial LIMS in support of the H3Africa biospecimen collections. Commercial LIMS are much more expensive upfront than open-source LIMS, are less flexible for end-user adaptations, but do not need local expertise for support. Despite some features of modified open-source LIMS that might seem more applicable to the African setting, there were significant concerns about the stability of such systems and the lack of standardization. In addition, it was clear that the adaptation and maintenance of such a system would need highly specialized staff at three biorepositories and that this approach could create differences among the biorepositories, which could cause potential delays in interbiorepository transfer of data and material.

\section{Financing of LIMS in the LMIC biorepositories}

The functioning of biorepositories requires stability and continuity of the LIMS. In addition to the buying of commercial licenses, some unforeseen expenses were encountered in the implementation of the commercial LIMS in H3Africa included training costs for staff and more complex hardware requirements, unlike for open-source LIMS. In H3Africa, it was possible to budget for these contingencies, but these costs may become prohibitive for smaller biorepositories outside of a funded mechanism. In such cases, potential creative solutions include forming consortia with similar facilities to buy a multiuser license. This approach has the added advantage of enabling a shared forum for dealing with other problems. However, since each biorepository has separate views, specific role-based security would be implemented for each user's collections. It may also only be necessary to acquire specific modules within a commercial package to keep the costs lower.

Retaining and training staff to use LIMS have presented some challenges for H3Africa. Some strategies were used, including actively searching for personnel with experience, ensuring that the LIMS manager feels integrated into the biorepository management structure, and training junior staff to make sure that there is an adequate succession plan. In addition, including training clauses within purchasing agreements have mitigated some of the risk of purchasing complex licenses, but ongoing training represents an essential need and should be part of a biorepository's quality management system. Discussion of formulating in-house training material for 
Table 1. Summarized Checklist to Consider While Choosing a Biorepository

LABORATORY INFORMATION MANAgEMENT System

LIMS general information

- What development tools have been used in the creation of the LIMS (e.g., Microsoft C\#)?

- Is the system scalable and therefore suitable for small organizations as well as global corporations?

- What are the system requirements?

- Does the system allow concurrent operation of three or more database groups in the standard system?

System configuration/customization

- Is your system configurable so that it can exactly meet our requirements? If yes, what are the requirements? If no, what are the alternatives?

- Is the system configurable by the nonprogrammer and without use of special languages?

- Are user configuration changes supported by the vendor? Is there any extra cost?

User access/security

- What is the security access system in detail?

- Does your system password requirements comply with 21CFR Part 11 ?

Functionality—sample/work registration

- Does your system support the following types of registration: Single sample; Single sample with copy feature; Batch registration with/without copy feature; Bar-code support; Registration templates; Registration from external system and/or scheduler; Spreadsheet style registration, including data capture from Excel; Can details of submitters and sample types be viewed from the registration screen; Can reports (e.g., worksheets and labels be automatically generated)

- Does system allow fields to be populated automatically by defining a default value within the system which can be overtyped by a user with suitable authority?

Functionality_-sample receipt

- Is a sample receipt function provided with the system? Useful when some work is preregistered before the availability of the samples. The receipt function is needed to track the arrival of the samples. Useful in checking whether all expected samples arrive in the laboratory and the time interval between registration and receipt for each sample can be measured. The sample turnaround time in the laboratory should be measured from the receipt date and time.

- Does sample receipt allow for single sample, multiple sample, batch sample, and global sample receipt utilizing bar-codes where needed?

Functionality-sample preparation

- Is a sample preparation function provided with the system? This would be used to indicate that samples must complete the preparation stage before they are ready for testing.

- Does sample preparation allow for single sample, multiple sample, batch sample, and global sample preparation?

Functionality-result entry

- Does system include: Result entry by sample — entry of any/all test results for a single sample; Result entry by test-entry of one test result for multiple samples; Result entry for multiple samples and multiple tests in spreadsheet style; Result import from a variety of sources including files and instruments; Viewing of results previously entered using same selection criteria as for selection of samples for result entry; Viewing of test status.

Reporting

- What reporting tools does the system support?

- Is event triggered reporting-reports generated by sample status change, for example-included?

System support

- If I have a support question can I telephone a help-desk and immediately talk to a technical person familiar with my system? If yes, where would this person be located and what time can I call them? If no, what support scheme is in place?

- Does the vendor have global coverage if relevant? If so what are the support center locations?

- Are new version upgrades supplied to customers at no cost?

- How much effort is typically needed for the implementation of an upgrade and what do I have to pay?

- Will an upgrade preserve my configuration or custom code as well as my data?

- Do you guarantee that we will always be able to upgrade to the next version?

Miscellaneous

- Does system allow storage of BLOB files, for example, pictures and documents (Consent forms) associated with a sample or a test?

- Can a document management capability be fully integrated within the system?

- Describe the sample tracking features of your LIMS. How is this used to monitor inventory for example?

- Can I have separate databases for different departments within my organization (they may be in different locations)? Can these databases be configured differently? 
LIMS support is ongoing across all three H3Africa Biorepositories for them to stay aligned with training and SOPs.

\section{IT infrastructure}

In addition to high acquisition costs, commercial LIMS requires an IT infrastructure to fully support their function. IT infrastructure is a combined set of the hardware (e.g., servers and computers), software (e.g., operating software), and network systems required to deploy and support the LIMS. As biospecimen numbers grow, there is a corresponding decrease in LIMS functionality such as very slow loading and processing speeds, which is linked to the supporting IT infrastructure. ${ }^{8}$ Therefore, a LIMS infrastructure should have the ability to be scaled to meet the needs of the community it serves. Institutions that are unable to support a biorepository LIMS on their own should team with other institutions to develop and deploy a shared biorepository LIMS infrastructure. By using a shared infrastructure, each participating institution could support components of the infrastructure independently, while also collectively managing the entire biorepository architecture. This will not only give an economical benefit but also offers an environment for harmonizing complex, but still critical, components of a LIMS, such as structured data files and data models, as well as standards for data transmission.

\section{User support services}

Commercial LIMS often needs user support services such as customization, implementation assistance, annual licenses, maintenance, and updates, although the need for these services may vary over time especially as users become experienced with the system. User support can be provided in several forms, including telephonic support and on-site support. There is an additional cost to get access to such services, which may be minimized by conducting thorough initial training and license negotiation. Many commercial LIMS vendors have no support networks or offices in Africa. This increases the costs of user support services because of airfare, accommodation, and other attendant costs to access the services. In such circumstances, African biorepositories should try to use other remote-access technology to get access to support, such as public IP addresses that enable external access and manipulation of the LIMS. Unlike commercial LIMS, open-source LIMS do not have user support services and the user must troubleshoot locally, which can be challenging or impossible based on technical capacity.

\section{LIMS harmonization in the H3Africa biorepositories}

Before the H3Africa program, each biorepository had its own LIMS that met their current needs. Since the commencement of the program, the biorepositories have either acquired new LIMS or upgraded their existing systems following a thorough LIMS assessment program. The biorepositories conducted a harmonization exercise to ensure interoperability of the LIMS across the three sites. Pilot studies conducted between H3Africa biorepositories integrated data sharing and importation protocols through a pilot biospecimen and data exchange. Data exchange harmonization is essential if biorepositories are to work efficiently in networks such as H3Africa to support population genomics studies. Biorepositories need to define an agreed sharable set of data and data format for harmonization and interoperability to ease exchange. Pilot projects with virtual data transfer protocols were undertaken successfully, suggesting that this harmonization has been effective.

\section{LIMS sustainability}

The biorepositories should develop strategies for longterm LIMS sustainability. Reliable and adequate sources of funding are keys to sustainability of LIMS. Commercial LIMS vendors require the payment of annual support fees unlike open-source LIMS. The risk of liquidation needs to be considered as it impacts LIMS support and maintenance and the long-term viability of the biorepository. Biorepositories should implement cost recovery measures for users to ensure sustainability.

\section{Conclusion}

Developing a state-of-the-art biorepository requires much capacity and staff development, including acquisition of formal training, equipment, and software. Key among the biorepository infrastructure needs is a LIMS. Choosing a LIMS in low- and middle-income countries requires careful consideration of the various factors that could affect its successful and sustainable deployment and use. H3Africa biorepositories operating in a consortium have highlighted key factors that affect recommendations for successful LIMS choice and implementation.

\section{Acknowledgments}

The H3Africa Consortium Biorepositories are funded by the U.S. National Institutes of Health grants UH3HG007008, UH3HG007051, UH3HG007438, and UH2HG007092 of the H3Africa Common Fund Initiative. The authors thank the Autoscribe Informatics (Berkshire, UK) that provided the Laboratory Information Management System (LIMS) User Checklist used in the LIMS evaluation process. The following Investigators were members of the H3Africa Biorepository PI Working Group: Jennifer Troyer and Louise Wideroff, National Institutes of Health, Washington, District of Columbia. Sue Penno, Biorepository Consultant, National Institutes of Health, Washington, District of Columbia. Christine M. Beiswanger, Coriell Institute for Medical Research, Institute of Human Virology-Nigeria, Abuja, Nigeria.

\section{Author Disclosure Statement}

No conflicting financial interests exist.

\section{References}

1. Rotimi C, Abimiku A, Kyobe S, et al. Research capacity. Enabling the genomic revolution in Africa. Science 2014; 344:1346-1348.

2. H3Africa Working Group. Harnessing Genomic Technologies Toward Improving Health in Africa: Opportunities and 
Challenges, in H3Africa White Paper. Washington, DC: National Institutes of Health; 2011.

3. Campbell LD, Betsou F, Garcia DL, et al. Development of the ISBER Best Practices for repositories: Collection, storage, retrieval and distribution of biological materials for research. Biopreserv Biobank 2012;10:232233.

4. Vaught J, Campbell LD, Betsou F, et al. The ISBER Best Practices: Insight from the editors of the third edition. Biopreserv Biobank 2012;10:76-78.

5. Pitt K, Betsou F. The ISBER Best Practices Self Assessment Tool (SAT): Lessons learned after three years of collecting responses. Biopreserv Biobank 2012;10:548549 .

6. Betsou F, Luzergues A, Carter A, et al. Towards norms for accreditation of biobanks for human health and medical research: Compilation of existing guidelines into an ISO certification/accreditation norm-compatible format. Qual Assur J 2007;11:219-292.
7. Landgraf KM, Kakkar R, Meigs M, et al. Open-source LIMS in Vietnam: The path toward sustainability and host country ownership. Int J Med Inform 2016;93:92-102.

8. Henricks WH. Laboratory information systems. Clin Lab Med 2016;36:1-11.

Address correspondence to: Samuel Kyobe, MBChB, MMed Micro Department of Medical Microbiology School of Biomedical Sciences Makerere University College of Health Sciences 4th Floor, Pathology/Microbiology BLDG

P.O. Box 7072

Kampala Uganda

E-mail: samuelkyobe@gmail.com; samkyobe@chs.mak.ac.ug 